\title{
Motor Nerve Terminal Sprouting in Formamide-Treated Inactive Amphibian Skeletal Muscle
}

\author{
M. M. Wines and M. S. Letinsky \\ Department of Physiology and Ahmanson Laboratory of Neurobiology, University of California, Los Angeles, Los Angeles, \\ California 90024-1751
}

\begin{abstract}
Motor axons can form sprouts from their terminal arborizations in response to partial denervation, and when exposed to pharmacological blocking agents like TTX, botulinum toxins $\alpha$-bungarotoxin, or curare. Each of these experimental procedures has cessation of muscle contractile activity as a common feature. We tested the specific role of muscle fiber inactivity in regulating nerve terminal sprouting by chronically treating adult frog (Rana pipiens) cutaneous pectoris muscles with formamide. Exposure to formamide, unlike the other compounds used to study sprouting, selectively inhibits muscle contractions without blocking pre- or postsynaptic transmission or muscle fiber action potentials. Repeated formamide applications were used to achieve chronic block of muscle contractile activity in vivo for up to 6 weeks. Motor axons in formamide-treated inactive muscle sprouted only from their terminal arborizations, but not from nodes of Ranvier. The onset of this sprouting was protracted compared with that seen in pharmacologically blocked mammalian muscles, and sprouts in formamide-treated muscles were more complex and ornate. The frequency of sprouting terminals was less in these formamide-treated muscles than that seen after alternate methods of contractile block, and this suggests that contractile inactivity alone serves as only a moderate cue for sprouting. The possibility is discussed that the prolific sprouting seen following neurotoxin administration may, in fact, be due to perturbations in synaptic transmission or muscle electrical activity rather than muscle fiber inactivity.
\end{abstract}

It has long been known that adult motor neurons are capable of expanding their field of innervation by forming new neuritic processcs, tcrmed sprouts (for reviews, see Edds, 1953; Brown et al., 1981; Grinnell and Herrera, 1981; Wernig and Herrera, 1986). These sprouts emerge from the intramuscular nodes of Ranvier and terminal arborizations of motor nerves. Although motor neuron sprouting was initially observed in striated muscles following partial denervation (Edds, 1950; Hoffman, 1950; reviewed by Edds, 1953; Brown et al., 1981), it has been seen

\footnotetext{
Received Nov. 19, 1987; revised Feb. 1, 1988; accepted Feb. 16, 1988.

We gratefully acknowledge G. Garrett, S. Carter, S. Specter, M. L. TeVrucht, and $C$. Higgins for their untiring help, and $H$. Kabe for expert photographic assistance. We also thank Drs. Y. Kidokoro, A. Herrera, and D. C. Linden for helpful comments on the manuscript. This work was supported by grants from the USPHS and Easter Seals Research Foundation. M.M.W. was supported by a USPHS Training Grant.

Correspondence should be addressed to Dr. Michael S. Letinsky, Department of Physiology, UCLA School of Medicine, Los Angeles, CA 90024-1751.

Copyright (C) 1988 Society for Neuroscience $0270-6474 / 88 / 103909-11 \$ 02.00 / 0$
}

more recently in response to spinalization (Brown et al., 1980b; Eldridge et al., 1981) or following application of a variety of nerve toxins that interfere with nerve conduction, transmitter release, postsynaptic response, or axoplasmic transport [e.g., botulinum toxin, tetanus toxin, TTX, or $\alpha$-bungarotoxin (Duchen and Strich, 1968; Duchen and Tonge, 1973; Brown and Ironton, 1977; Holland and Brown, 1980, 1981)]. Sprouting is also seen in diseased muscle (Rowe and Goldspink, 1969; Duchen, 1970; Coers et al., 1973; I Iarris and Ribchester, 1979) and in senescent animals (Barker and Ip, 1966; Tuffery, 1971; reviewed by Wernig and Herrera, 1986). All of these conditions that promote sprouting have muscle fiber denervation and/or inactivity in common. Further, the observation that frequency of sprouting in some cases can be significantly reduced by artificially restoring contractility (Brown et al., 1977; Brown and Holland, 1979; Brown et al., 1980b), coupled with the above results, strongly implicates muscle fiber inactivity as a stimulus to motor neuron sprouting. Despite the considerable evidence that inactive muscle fibers are, directly or indirectly (through subsequent denervation-like muscle fiber changes), a source of some sprouting factor(s) (reviewed by Brown et al., 1981; Brown, 1984), other interpretations of these and similar results have engendered different hypotheses (e.g., O'Brien et al., 1978; Pestronk and Drachman, 1978). However, the direct role of muscle fiber inactivity as a signal for motor nerve sprouting descrves investigation if we are to fully comprehend the signals that contribute to plasticity at the neuromuscular junction.

Terminal sprouting results whenever muscle fiber contractile activity is impaired by either partial denervation or poisoning with a neurotoxin. However, in each case different steps in the sequence from nerve activity to muscle contraction are blocked. Moreover, the sprouting motor nerve terminals are subjected to vastly different environments depending upon the experimental paradigm. Partial denervation, for example, produces a population of normally functioning motor neurons and innervated muscle fibers juxtaposed with inactive muscle fibers that have denervated postsynaptic specializations, degenerating axons, and degenerating nerve terminals. In addition, this terminal sprouting occurs from neuromuscular junctions that themselves are unperturbed. By contrast, neurotoxin poisoning blocks neuronal or synaptic function and thereby produces inactive muscle fibers with morphologically intact neuromuscular junctions. Thus, the toxin-blocked preparations possess sprouting nerve terminals that themselves have impaired pre- or postsynaptic function and that reside on muscle fibers having varying degrees of contractile inactivity.

Although muscle fiber electrical and mechanical inactivity is 
the end result of partial denervation or neurotoxin application, it is obvious that varied components of the neuromuscular system have been compromised to achieve this state. For example, application of a presynaptic neurotoxin not only removes transmitter release (and probably the release of any putative presynaptic trophic factor), but also eliminates the postsynaptic $\mathrm{ACh}$ receptor response and any subsequent muscle fiber electrical and contractile activity. Conversely, inhibitors such as $\alpha$-bungarotoxin spare presynaptic release but specifically inhibit postsynaptic $A C h$ receptor function and muscle fiber electrical and mechanical activity. Because of the inability of any of these methods to selectively inhibit a single physiological parameter, it is impossible to assess whether the primary cause of sprouting is muscle fiber inactivity alone or impaired neuromuscular function and/or the presence of denervation and degenerating nerve tissue. Further, these experimental approaches cannot provide insight into the role of contractile inactivity upon biochemical processes relevant to sprouting. To overcome this we have developed an experimental system in which muscle electrical and mechanical activity, as well as postsynaptic neuromuscular function, can each be blocked independently and their respective influence on sprouting can therefore be assessed. This is accomplished by chronically blocking muscle contractile activity with formamide (Wines et al., 1985). Repetitive formamide treatment produces a muscle preparation with intact neuromuscular innervation and functional synaptic transmission and muscle fiber electrical activity (action potentials) but without contractile activity (Escalona de Motta et al., 1982; Herrera, 1984). Thus, this preparation allows for a selective assessment of the stimulatory role of muscle contractile inactivity in producing motor nerve terminal sprouting.

\section{Materials and Methods}

Experimental preparation. The paired cutaneous pectoris (CP) muscles of adult Rana pipiens (5-7 cm body length) were chosen for this study. Experiments were performed throughout the period from August 1984 to February 1986. Each CP is a relatively thin quadrangular sheet of muscle that lies just beneath the pectoral skin. This superficial location makes the muscle relatively accessible, and repetitive surgical manipulations can therefore be performed without difficulty (Wines et al., 1985; scc also McMahan et al., 1980); in addition, its thin dimensions (3-6 muscle fibers thick) allows for clear visualization of nerve terminals for electrophysiological studies and subsequent histological analysis on whole-mount preparations (e.g., Letinsky et al., 1976; Morrison-Graham, 1983).

Chronic muscle inactivity was produced by repeated in situ applications of formamide (Kodak). Following anesthetization in $0.1 \%$ tricaine methanesulfonate (Sigma), frogs $(n=29)$ were chilled in a refrigerator at $4^{\circ} \mathrm{C}(5-10 \mathrm{~min})$. The animal was then positioned on a moistened towel atop a bed of crushed ice, and the entire preparation placed in the path of a stream of air cooled by dry ice (Fig. 1). This degree of cooling dramatically lowered the animal's heart rate and thereby reduced the amount of formamide entering the cardiovascular circulation. This protracted cooling was not harmful, and all animals recovered. A Ushapcd incision approximately $3.0 \mathrm{~cm}$ long was made to expose the entire right $\mathrm{CP}$ muscle, the adjacent lateral pectoralis muscle, and a portion of the contralateral $\mathrm{CP}$. The muscles were moistened with chilled normal frog Ringer's (NFR) solution (approximately $8^{\circ} \mathrm{C}$ ) containing $116 \mathrm{~mm} \mathrm{NaCl}, 2 \mathrm{~mm} \mathrm{KCl}, 1.8 \mathrm{~mm} \mathrm{CaCl}_{2}, 5.0 \mathrm{~mm}$ HEPES, pH 7.3. As shown in Figure 1, a watertight well $(\sim 1 \mathrm{ml})$ consisting of orabase gel (Hoyt Laboratories) was applied to the anterior abdominal wall and surrounding the exposed CP muscle (Tal and Rotshenker, 1984). After the integrity of the reservoir was checked, the well was filled with an experimental formamide solution containing salts equal to that of NFR. Following a 15-25 min incubation, the formamide solution was gradually diluted by the dropwise addition of chilled NFR solution for a period of $30 \mathrm{~min}(1 \mathrm{drop} / 8-10 \mathrm{sec})$. A 23 -gauge syringe needle attached to polyethylene tubing and connected to a vacuum line was positioned over the well to limit the level of the meniscus during the rinse (Fig. 1). After rinsing, the orabase was carefully removed without damaging adjacent tissues by directing a stream of NFR along the gel's interface with the body. The wound was closed by approximating the skin flaps with a pair of fine-tipped forceps while simultaneously applying a few drops of the surgical tissue adhesive Histoacryl Blue (B. Braun Melsungen $\Lambda G$, Tri-Hawk) along the incision (Fig. 1). With this technique the incision healed rapidly and cleanly, appearing as a thin line of connective tissue within a few days. Most importantly, this wound could be repeatedly opened (up to 10 times) by cutting along the original incision without the significant necrosis and progressive loss of healing skin that usually results from repeated closing with silk sutures. The entire operative procedure required about $90 \mathrm{~min}$, after which animals were placed on moist towels to recover from the anesthetic. Muscles were rendered chronically inactive for up to $42 \mathrm{~d}$ by repetitive treatments with formamide every $4 \mathrm{~d}$. Because consecutive treatments with $4.0 \mathrm{M}$ formamide caused severe muscle fiber damage, we established a protocol of bathing the muscle in varying concentrations of formamide. With this procedure contractile inactivity was maintained without significant structural damage. The incubation regimen involved the original $4.0 \mathrm{M}$ incubation, followed after $4 \mathrm{~d}$ by a second treatment of equal duration using a $0.5 \mathrm{M}$ formamide in the NFR salt solution. For longer periods of inactivity subsequent treatments were performed every $4 \mathrm{~d}$ using 0.75 and $1.0 \mathrm{~m}$ solutions, respectively, after which all further treatments utilized a $1.0 \mathrm{~m}$ solution. This schedule produced the most effective contraction block while still preserving muscle fiber integrity.

Assessing neuromuscular function. The treated and contralateral $\mathrm{CP}$ muscles were dissected out, and the experimental muscle was pinned to a Sylgard-lined petri dish that had been modified for electrophysiological recording (Morrison-Graham, 1983). Each preparation was observed (stereo microscope, $\times 25-50$; compound microscope, $\times 200$ $400)$ to determine the extent of the formamide block; nerve-evoked contractions in individual muscle fibers were easily detectable. In several experiments, both synaptic function and evoked muscle fiber action potentials were monitored. Nerve-evoked muscle fiber action potentials were recorded at junctional sites. For electrophysiological analysis, individual nerve terminals were observed with Nomarski optics, and conventional intracellular recording techniques were employed using glass microelectrodes filled with $0.6 \mathrm{M} \mathrm{K}_{2} \mathrm{SO}_{4}$. The nerve to the $\mathrm{CP}$ was stimulated via a small suction electrode (square pulses, $0.33 \mathrm{~Hz}, 200$ $\mu$ sec duration), and physiological events were collected with on-line computer facilities (Digital Micro 11/23). During this portion of the experiment the tissue was continuously perfused with fresh NFR.

Histology. The peripheral innervation of both the treated and the contralateral unblocked CP muscles was stained. Demonstration of the presynaptic nerve terminals was accomplished as follows. Muscles were pinned flat in petri dishes and immersed for $3 \mathrm{~min}$ in $0.09 \mathrm{M}$ carbonate buffer (pH 9.0) containing 2\% glutaraldehyde (fresh EM grade; Polysciences) and $3 \mathrm{mg} / \mathrm{ml}$ tetranitroblue tetrazolium salt (TNBT; Sigma). The preparation was then rinsed for $3 \mathrm{~min}$ in the buffer/fixative solution, followed by a $4 \mathrm{~min}$ incubation in the buffer/fixative solution with 0.3 $\mathrm{mg} / \mathrm{ml}$ 1-methoxyphenazine methosulfate (Polysciences). Postsynaptic acetylcholinesterase activity was demonstrated according to methods outlined by Karnovsky (1964; see also Letinsky and DeCino, 1980; Letinsky, 1983) and the myelinated portion of the motor neurons were poststained for 5 min with $0.3 \%$ aqueous $\mathrm{OsO}_{4}$ (Polysciences) in phosphate buffer ( $\mathrm{pH}$ 7.2). Muscles were then mounted whole between coverslips in Aquamount (Lerner Laboratories). Nerve terminal structure and terminal sprouting were observed using Zeiss 40/1.0,63/1.4, and 100/ 1.3 planapochromatic objectives. These high-resolution objectives are necessary to clearly detect fine $(<0.5 \mu \mathrm{m})$ outgrowths emerging from the nerve terminal. Observations are further facilitated by the color contrast between the purple formazan-stained neural processes, the Hatchett's brown Karnovsky reaction product, and the yellow muscle fibers. Between 27 and 92 nerve terminals on the most superficial muscle fibers were analyzed in each CP muscle. A 2-tailed Student's $t$ test was used to assess the significance of the results.

\section{Results}

\section{Formamide block of contractile activity}

The effect of formamide on muscle fiber contractions was dramatic. A single $4.0 \mathrm{M}, 20 \mathrm{~min}$ in situ treatment generally rendered the CP muscle $90-100 \%$ blocked for at least $4 \mathrm{~d}$ and sometimes up to 5-7 d. The effectiveness of this block was 


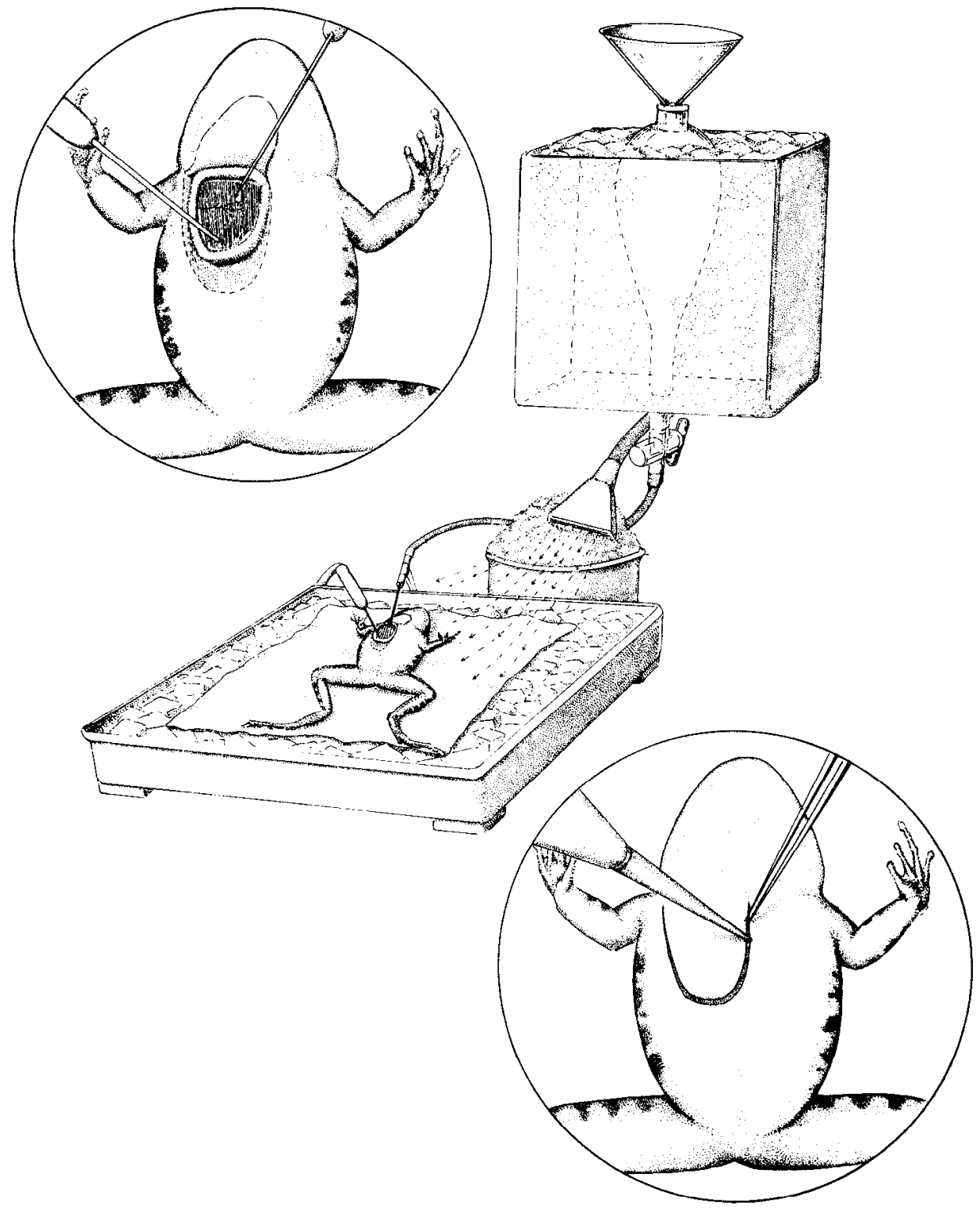

Figure 1. Illustration of the experimental protocol demonstrating the position of the orabase reservoir relative to the inflow and outflow tubes (upper inset), the general arrangement of the setup during the formamide incubation (center), and the method of closure of the surgical wound (lower inset). primarily dependent upon the penetration of formamide; residual contractions usually appeared only at the ends of the muscle fibers, where the access of formamide was restricted because of extensive connective tissue and overlying tissucs. In gcncral, the block was always more effective in the central portions of the muscle where each fiber was fully exposed to formamide. The efficacy of the contractile block was seen to diminish with time. The return of function first occurred among the smaller-diameter muscle fibers located along the muscle's medial edge (see Letinsky and Morrison-Graham, 1980; Linden and Letinsky, 1983, 1988); subsequently, contractile activity relurned throughout the remainder of the muscle. To achieve uninterrupted chronic block of muscle contractions we therefore repeated the in situ formamide application every $4 \mathrm{~d}$.

Repetitive exogenous application of formamide proved to be effective in eliminating CP muscle contractile activity in these experiments for up to $42 \mathrm{~d}$. In half of the dissected preparations indirect nerve stimulation evoked either no visible contractions or contractions of only a very small number of muscle fibers (approximately 1-10 fibers typically located along the muscle's medial border). The balance of the experimental preparations showed somewhat more twitch, with several muscles $(n=6)$ demonstrating more than $20-25 \%$ twitch. These active fibers were typically located within the medial third of the muscle, with most of the contractile activity confined to the tendon ends of the muscle fibers. We confirmed the location of the twitch, which sometimes occurred in individual muscle fibers. These contractions often occurred at the tendinous ends, where, because of connective tissue and diffusion barriers, the fibers were less well exposed to formamide and thus less well blocked. This twitching was eliminated by selectively crushing the active fibers with fine forceps approximately $0.5-1.0 \mathrm{~mm}$ from their tendinous ends. Such crushing eliminated much of the twitching and confirmed that the central endplate regions of the CP muscle, which were fully exposed to formamide, were completely inactive. Thus, any activity in this region was the result of passive stretching rather than active shortening. Since visual inspection was the method used to assess the amount of twitch, it was 


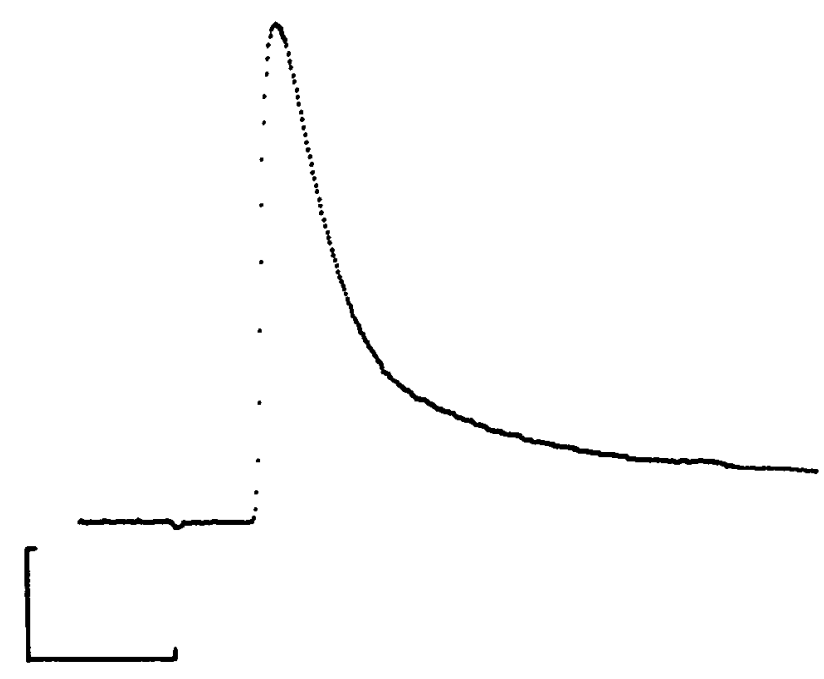

Figure 2. Intracellularly recorded muscle fiber action potential from a $5 \mathrm{~d}$ formamide-treated preparation. The recordings were made at $18^{\circ} \mathrm{C}$. Scale bars, $20 \mathrm{mV}$ rise, $5 \mathrm{msec}$.

impossible to make a quantitative determination of the absolute degrce to which contractile activity was removed. However, even in those muscles demonstrating incomplete block, it was apparent that a significant reduction in contractile activity had occurred. Further, it was also clear from our observations that in all of the treated muscles superficial fibers remained completely inactive and that any observed twitch was the product of contracting fibers at deeper planes within the muscle, thus suggesting that the effectiveness of the formamide block is dependent on its penetration.

Interestingly, in situ formamide application produced less muscle fiber damage and a more effective contractile block compared with in vitro formamide application (Escalona de Motta et al., 1982; Herrera, 1984). The general integrity of muscle fibers exposed to formamide in situ was quite good, with light microscopy confirming normal striations and fiber profiles. In the few instances when muscle fiber damage was present, it was easily detected by light microscopy and initially appeared as a generalized "pitting" of the muscle surface.

The general proliferation of connective tissue that often accompanies repeated opening and closing of a surgical wound was insignificant in these preparations, the small amount occasionally developing on the muscles' superficial surface being easily removed with fine-tipped forceps.

\section{Neuromuscular function}

Conventional intracellular recording methods were employed in 6 experimental muscles exposed to formamide for $1-5 \mathrm{~d}$ ( 1 or 2 treatments). Formamide incubation had no deleterious effect on functional neuromuscular physiology. Suprathreshold endplate potentials and resulting action potentials (Fig. 2) were recorded at 118 out of 127 neuromuscular junctions, and those junctions without action potentials had large subthreshold epps. Further evidence of functional neuromuscular transmission was verified by the presence of nerve-evoked muscle fiber contractions occasionally observed during the course of these experiments. In addition, the resting frequency of miniature endplate potentials was within the normal range ( $1-5 \mathrm{mepps} / \mathrm{sec})$, as was the average resting potential $(79-91 \mathrm{mV})$. We conclude, therefore, that chronic formamide treatment as applied in these ex- periments, while rendering CP muscle fibcrs mechanically inactive, spares synaptic potentials and muscle fiber action potentials.

\section{Motor nerve response to chronic inactivity}

A search for anatomical changes in axons and motor nerve terminals in response to chronic formamide induced muscle fiber inactivity showed that molor nerve terminals survived repeated formamide application well. TNBT/Karnovsky AChEstained neuromuscular junctions appeared normal (see Letinsky and DeCino, 1980), and we found no light microscopic evidence of muscle fiber denervation, nerve regeneration, or nerve terminal disruption. However, we did find evidence of nerve terminal growth, which corresponded to terminal sprouting. Motor neurons within formamide-treated muscles preferentially produced terminal sprouts; nodal sprouting was never observed. Since there are differences in the sprouting nomenclature, we defined a terminal sprout as any unmyelinated TNBT-stained outgrowth originating from a nerve terminal process and extending beyond the boundaries of postjunctional acetylcholinesterase activity as revealed by the Karnovsky reaction product (Fig. 3). Thin, varicose nerve processes that had associated AChE activity, but that remained on their parent muscle fiber (e.g., ring fibers or jumping fibers as defined by Wernig et al., 1980), were not considered to be sprouts. Therefore, our definition of terminal sprouts only applied to presumably new terminal outgrowths or to sprouts that had not formed synaptic contacts as evidenced by associated postsynaptic AChE activity.

We determined the extent of sprouting of well-stained, clearly visible superficial nerve terminals. Sprouting motor nerve terminals were observed throughout the muscle, and there was no apparent grouping or clustering of sprouting terminals. There was also no correlation between nerve terminal size or complexity and sprouting. The frequency of sprouting was relatively small during the first week of contractile inactivity, with only $6-12 \%$ of the terminals within the muscle bearing sprouts (Table 1, Fig. 4A). A slight increase in the number of sprouting terminals was noted after 2 weeks of sustained inactivity, with on the average only $25 \%$ of the observed endplates seen to sprout at this timc. Tablc 1 clearly shows that a broad range of sprouting frequencies occurred throughout the experiment's duration. For example, for 3 animals in which the C.P had been inactive for a period of $14 \mathrm{~d}$, the range of sprouting frequencies was between $8-46 \%$. There was a significant correlation $(R=0.43, p<0.05)$ between lengthening periods of contractile inactivity and the number of sprouting terminals.

With regard to sprout morphology, light microscopy of motor nerve terminals in treated muscles showed an array of sprout profiles and lengths (Fig. 5). Short sprouts $(1-10 \mu \mathrm{m})$ extending parallel to the parent muscle fiber and terminating as a small varicosity were most common. Moreover, there was no significant correlation between the duration of inactivity and sprout length $(R=-0.27, p>0.05$; Fig. $4 B$ ). Over the course of the experiment, more ornate sprouts were also occasionally present (Fig. 6). These complex sprouts often had multiple sites of bifurcation accompanied by intermittent swellings along their length (up to $250 \mu \mathrm{m}$ ). Directed growth of these elaborate sprouts either across or along the muscle fiber length was never observed; histologically, these sprouts did not appear to form synaptic contacts with adjacent muscle fibers as postjunctional staining of acetylcholinesterase (Letinsky and Morrison-Graham, 1980) beneath the sprouts was never observed. However, 


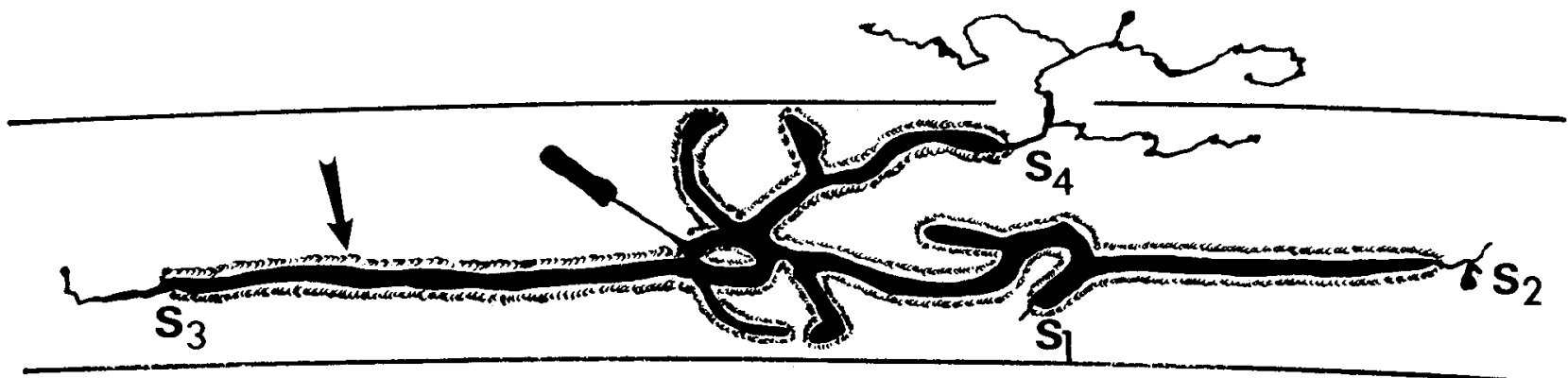

Figure 3. Schcmatic illustration depicting a neuromuscular junction with its acetylcholinesterase reaction product ( 4$)$ and 4 terminal sprouts with varying degrees of complexity (S1-S4). The extension of the neurites beyond the postsynaptic enzyme reaction product classifies them as terminal sprouts. Sprouts of this morphology were considered in our analysis.

our measurement of sprouting frequency may be an underestimate since more mature sprouts could have induced acetylcholinesterase and therefore would not have been included in our analysis.

Muscles were also examined for other responses to formamide treatment. An additional interesting observation was that unmyelinated axons proliferated in some treated muscles. How- ever, this was not a regular occurrence, and further attempts to quantify these changes were not made. Last, as shown in Table 1 , sprouting in the contralateral untreated $\mathrm{CP}$ was insignificant.

\section{Control experiments}

The nerve terminal sprouting that occurs in response to chronic formamide-induced muscle inactivity might be initiated by the
A

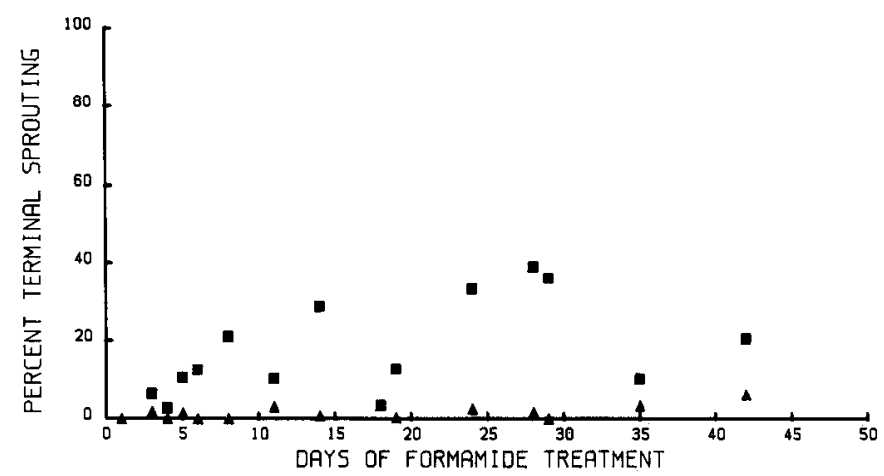

B

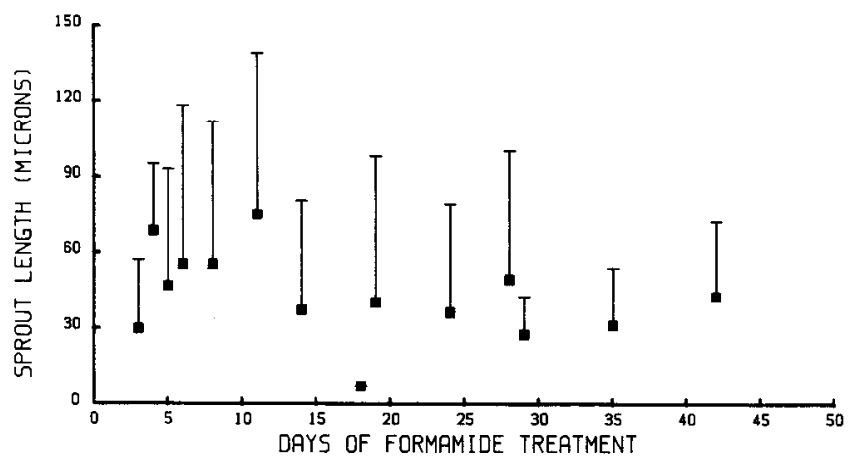

Figure 4. Graphs plotting the frequency of terminal sprouting $(A)$ and the length of terminal sprouts $(B)$ against the number of days of chronic formamide treatment. Symbols: $\mathbf{\square}$, formamide-treated CP muscles; $\square$, untreated contralateral CP muscles. (SDs of the average sprout length are presented only in the positive direction for clarity.) 

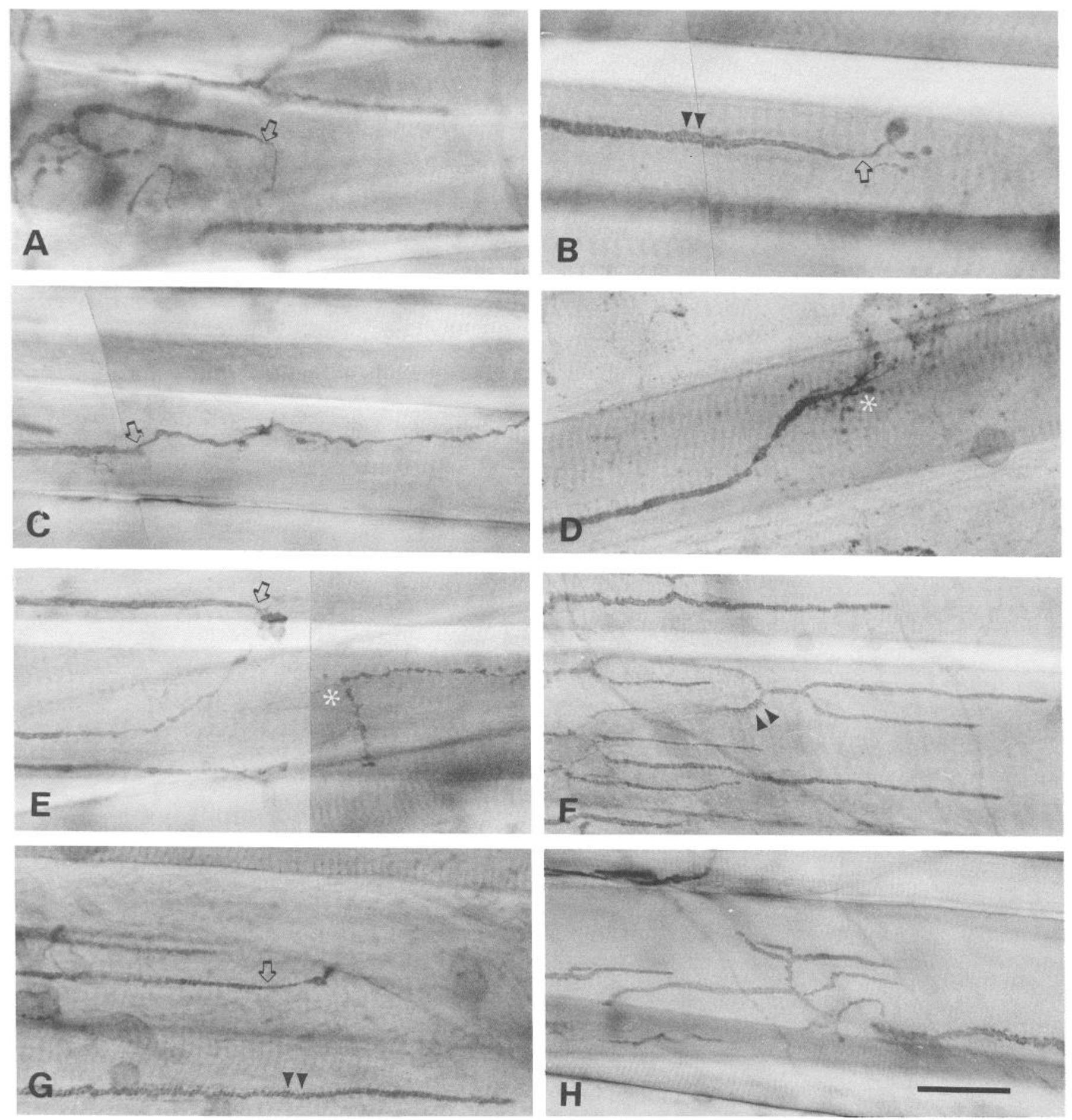

Figure 5. Examples of TNBT-stained nerve terminals exhibiting a variety of sprout morphologies following formamide incubations of $(A) 5$, $(B$ and $C) 14,(D) 19$, and $(E) 29 \mathrm{~d}$. The arrow $(D)$ marks the location of sprout origin. Notice the growth of the sprouts beyond the lightly stained AChE reaction product $(\downarrow)$ in $B$ and $G$. Note also the structural complexity of a number of sprouts (asterisk in $D$ and $E$ ). The remaining panels show examples of nonsprouting terminals within a preparation after $19 \mathrm{~d}$ of formamide treatment $(F)$; and sprouting and nonsprouting terminals from untreated contralateral CP muscles at $19 \mathrm{~d}$ ( $G$ and $H$, respectively). Scale bar, $20 \mu \mathrm{m}$ in $B, 50 \mu \mathrm{m}$ in $F$ and $H$, and $30 \mu \mathrm{m}$ in the remaining panels.

formamide itself or by the experimental procedures. Several series of experiments were devised to examine these possibilities.

To control for any direct sprout-promoting properties of formamide, we attempted to incubate the muscles in a manner that did not block muscle contractions. However, muscle con- tractions were always inhibited to varying degrees when the original formamide concentrations were applied even for less time. In order to expose muscles to formamide without rendering them inactive, $5 \mathrm{CP}$ muscles were treated with the usual $4 \mathrm{~d}$ regimen; however, the concentration of each formamide solution was diluted to $25 \%$ of its original strength and the 

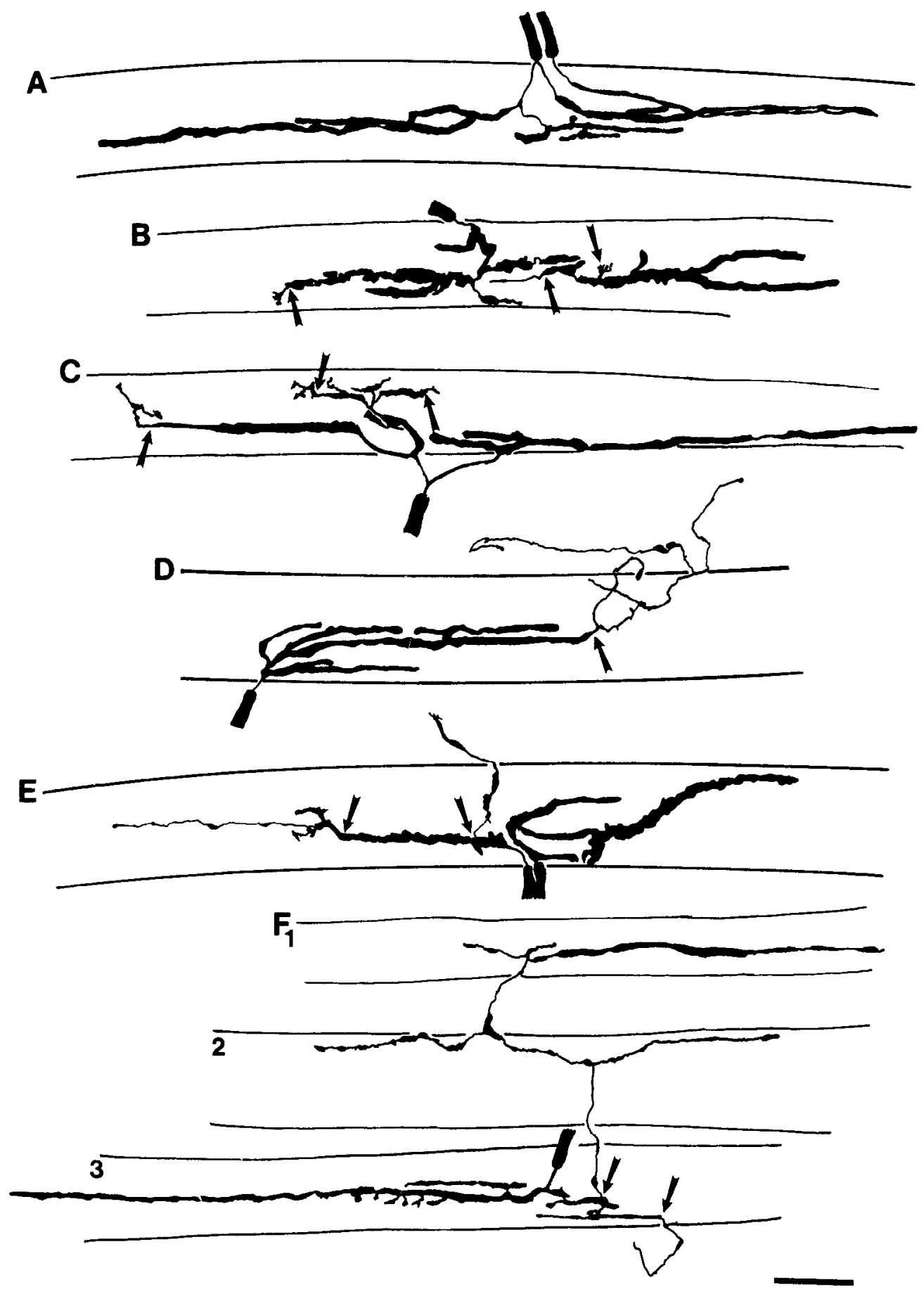

Figure 6. Camera lucida drawings of TNBT-stained motor neurons showing moderate and extensive arrays of sprouting after formamide treatment $(B$ and $C$ ) or partial denervation $(F)$. Note the ornate pattern of neurite division at $19(B$ and $C)$ and $14 \mathrm{~d}(D)$ posttreatment and the ability of sprouts to traverse adjacent muscle fibers $(D-F)$. A nonsprouting terminal from a formamide muscle $(A, 19 \mathrm{~d})$ is supplied for comparison. Arrows $(\rightarrow)$ show the site of sprout origin. Scale bar, $20 \mu \mathrm{m}$. incubation time was increased 4-fold (e.g., the first treatment utilized a $1 \mathrm{M}$ solution for $80 \mathrm{~min}$ versus $4 \mathrm{M}$ for $20 \mathrm{~min}$ ). Since each muscle presumably was exposed to an amount of formamide comparable to that which would normally have blocked contractions, extensive sprouting under such circumstances would support a direct formamide effect. However, all 5 muscles maintained for 21-24 d under this protocol exhibited very limited amounts of sprouting ( $0-4 \%$; Fig. $7 E$ ). However, these control experiments do not preclude the possibility that sprouting occurs in this model in response to a concentration-dependent mechanism.

In addition to controlling for the possibility that formamide might inherently induce sprouting, it was also pertinent to evaluate whether this compound suppresses neuronal growth. If the latter were true, then the observed motor neuron sprouting in response to muscle inactivity would be inhibited to some degree due to the presence of formamide. Thus, the effectiveness of muscle inactivity on nerve terminal sprouting would be underestimated. This possibility was tested for by carrying out the standard formamide protocol on CP muscles that were either totally denervated and allowed to reinnervate or were partially denervated to induce terminal sprouting. In the former case, the crushed CP nerve regenerated and reinnervated the muscle normally, suggesting that formamide treatment did not hinder nerve 

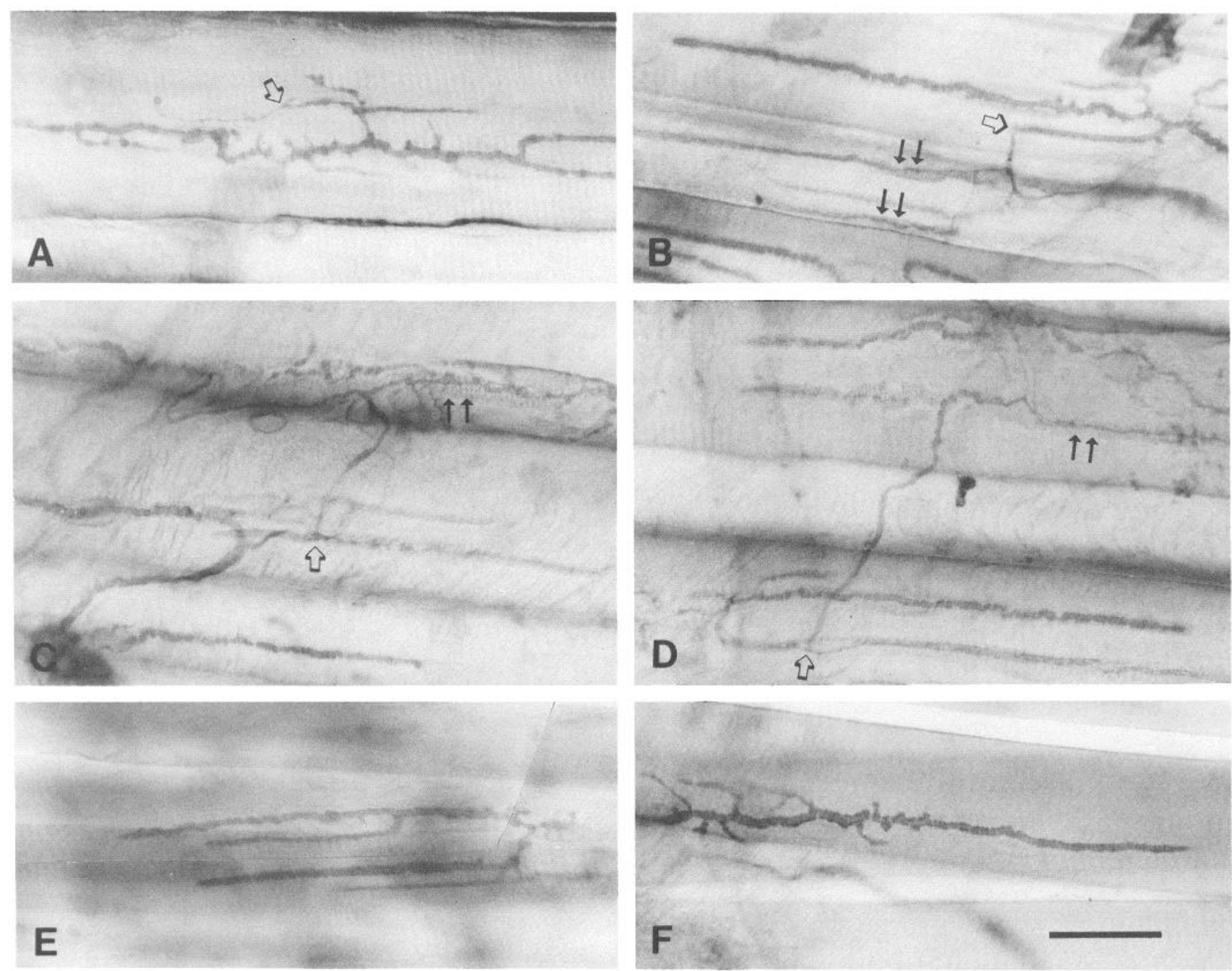

Figure 7. Motor neuron sprouting at $(A) 7$ and $(B) 12 \mathrm{~d}$ after partial denervation alone and at $14 \mathrm{~d}(C$ and $D)$ following partial denervation and ongoing formamide treatment. The remaining panels demonstrate nonsprouting endplates in control $(E)$ and formamide-treated $(F) \mathrm{CP}$ muscles. The open arrows $(\square)$ show the sites of sprout origin, while the solid arrows $(\Delta)$ mark the locations of sprout growth into adjacent empty postsynaptic "gutters." Scale bar, $30 \mu \mathrm{m}$.

growth or synapse formation. If formamide inhibited sprouting, then this effect would be easily detected in a formamide-treated, partially denervated muscle, where sprouting is known to occur.

Partial denervation of the $\mathrm{CP}$ muscle was accomplished by carefully severing approximately one-half of the $\mathrm{CP}$ innervation in spinal root 2 as it passes through the shoulder region. At this point, the nerve root is accessible, and we were able to reliably remove $30-60 \%$ of the innervation to the CP. The extent of partial denervation is easily determined anatomically once the muscle is stained and examined by light microscopy. Prolific terminal sprouting is evident as early as $7 \mathrm{~d}$ postoperatively (see Figs. 6 and 7), and sprouting is extensive throughout the muscle by $14 \mathrm{~d}$. In these control studies, $14 \mathrm{~d}$ after the onset of both partial denervation and simultaneous formamide treatments, motor nerve terminals in 5 muscles demonstrated copious sprouting from their terminal arborizations. These sprouts consistently grew toward adjacent denervated junctional sites, and the sprouts ramified through the empty postjunctional "gutters" (Fig. 7). In addition, both the morphology and frequency of sprouting in these "double-treated" muscles was comparable to that seen after only partial denervation. Thus, we have found no evidence that formamide inhibits nerve terminal sprouting.

We also controlled for the possibility that sprouting was a response to surgical manipulation and/or application of the orabase gel. In 3 animals the CP was exposed, and half of its superficial surface was covered with the gel. After a $15 \mathrm{~min}$ treatment, the gel was removed, the muscle rinsed, and the incision closed in our usual manner. Histological observation again showed very little sprouting, with 2 of the preparations demonstrating no sprouting after $7 \mathrm{~d}$, and the remaining muscle showing only $4 \%$ terminal sprouting after $14 \mathrm{~d}$. In addition, when 2 animals were treated as above for $14 \mathrm{~d}$ but with formamide replaced by NFR, there was also minimal sprouting ( 6 and $7 \%$ ).

One final consideration was that formamide treatment did not induce de novo sprouting but that we were merely measuring normal seasonal variations in terminal sprouting (see Grinnell and Herrera, 1981; Wernig and Herrera, 1986). Fourteen pairs 
of CP muscles [i.e., 12 muscles from summer frogs ( $\Lambda$ ugust) and 16 muscles from winter frogs (January and February)] from normal stock animals were examined for sprouting. The frequency of sprouting was $2 \pm 3 \%$ and $0.3 \pm 0.7 \%$ (means \pm SD) in summer and winter $\mathrm{CP}$ muscles, respectively. Within the constraints of our definition of terminal sprouting, these results show that there was no significant $(p>0.05)$ seasonal variation.

\section{Discussion}

As mentioned previously, there is abundant evidence to suggest that neuromuscular junctions can undergo significant structural and functional remodeling in response to both natural and experimental perturbations (reviewed by Brown et al., 1981; Grinnell and Herrera, 1981; Wernig and Herrera, 1986). The observation of motor neuron sprouting in response to partial denervation is the classical example of such synaptic plasticity. It has been postulated that the induction of this sprouting may occur in response to the presence of either (1) nerve degeneration products, noninnervated junctional sites, denervated muscle fibers or (2) as a product of the resultant contractile inactivity (Brown et al., 1981; Brown, 1984). Sprouting analogous to that seen in partially denervated muscles has also been noted within inactive muscles that have been pharmacologically treated to inhibit nerve conduction (Brown and Ironton, 1977), presynaptic transmission (Duchen and Strich, 1968; Duchen and Tonge, 1973), or postsynaptic response (Holland and Brown, 1980). Thus, sprouting can be seen to occur in muscles rendered inactive by either morphological changes such as the ablation of motor neurons or in response to alterations in physiological parameters such as synaptic transmission.

It was the aim of this study to develop a neuromuscular preparation in which contractile inactivity could be induced and maintained without damaging motor neurons or altering preor postsynaptic function and to further assess whether motor neurons given this unique environment would sprout. This was accomplished through the application of formamide upon mature amphibian muscle, which produced a specimen that was not only mechanically inactive, but that showed no deleterious physiological changes.

Sprouting occurred in a small proportion of nerve terminals in response to maintained contractile inactivity caused by repeated formamide application. The preferential terminal sprouting response that was observed (muscles were devoid of nodal sprouting) is analogous to that seen after toxin treatment in mammalian muscles (Duchen and Strich, 1968; Brown and Ironton, 1977). However, the onset of terminal sprouting following formamide treatment is markedly slower by approximately $10 \mathrm{~d}$, and the average frequency of sprouting is far less robust by comparison (Duchen and Strich, 1968; Brown and Ironton, 1977; Holland and Brown, 1980). Interestingly, the formamide-induced sprouting response is similar to one aspect of the response seen in amphibians following contractile inactivity produced by chronic curare treatment (Werniget al., 1980). In this study, 2 categories of sprouting were observed: (1) sprouts without apparent contact and (2) more elaborate sprouts with one or more specialized synaptic contacts. The more rudimentary of these sprout types, those lacking apparent synaptic contact, are commonly seen after both curare or formamide treatment and most likely represent the initial stages of sprout formation. It is interesting that a common sprout type occurs despite that each agent produces inactivity via a different mechanism: For example, curare inhibits synaptic potentials (epps)

\begin{tabular}{|c|c|c|}
\hline \multirow{2}{*}{$\begin{array}{l}\text { Days of } \\
\text { inactivity }\end{array}$} & \multicolumn{2}{|c|}{ Terminal sprouting (\%) } \\
\hline & Treated CP & Untreated CP \\
\hline 3 & 5 & - \\
\hline 3 & 7 & 2 \\
\hline 4 & 3 & 0 \\
\hline 4 & 2 & 0 \\
\hline 5 & 14 & 4 \\
\hline 5 & 8 & 2 \\
\hline 5 & 13 & 0 \\
\hline 5 & 8 & 0 \\
\hline 6 & 21 & 0 \\
\hline 6 & 4 & 0 \\
\hline 8 & 21 & 0 \\
\hline 11 & 3 & 2 \\
\hline 11 & 0 & 7 \\
\hline 11 & 28 & 0 \\
\hline 14 & 9 & 2 \\
\hline 14 & 21 & 0 \\
\hline 14 & 46 & 0 \\
\hline 18 & 3 & - \\
\hline 19 & 18 & 1 \\
\hline 19 & 7 & 0 \\
\hline 19 & 5 & 0 \\
\hline 19 & 21 & 0 \\
\hline 24 & 33 & 3 \\
\hline 28 & 39 & 2 \\
\hline 29 & 20 & 0 \\
\hline 29 & 53 & 0 \\
\hline 35 & 10 & 3 \\
\hline 42 & 20 & 4 \\
\hline 42 & 21 & 9 \\
\hline
\end{tabular}

and nerve-evoked muscle action potentials, whereas formamide treatment spares neuromuscular transmission and muscle fiber action potentials (Escalona de Motta et al., 1982; Herrera, 1984). This observation suggests that muscle fiber contractile inactivity alone (i.e., blocking at one of the biochemical steps leading to cross-bridge interaction and contraction), even in the presence of muscle fiber action potentials and synaptic transmission, appears sufficient to cause at least some motor nerve terminals to sprout.

The mechanism whereby inactive muscle fibers induce even a moderate terminal sprouting response is not yet clear. It has been suggested previously that inactive muscle fibers secrete a substance which directly (Betz et al., 1980; Slack et al., 1983; Brown, 1984; Gurney et al., 1986) or indirectly [i.e., through changes in membrane or surface molecules (Brown et al., 1980a)] induces nerve terminals to sprout. Indirect evidence for a muscle-derived factor is strongly implicated by the observation that amphibian nerve terminals fail to sprout even after months of residing on muscle fiber-free basal lamina ghosts (Yao and McMahan, 1985; McMahan, personal communication). More direct evidence for muscle-derived motor neuron growth factors has been suggested (Slack et al., 1983; Brown, 1984), and a potential sprout-promoting factor derived from denervated skeletal muscle grown in culture has been described (Gurney, 1984; Gurney et al., 1986). If this factor were a diffusible substance with only a limited range of effectiveness (Betz et al., 
1980; Slack and Pockett, 1981, 1982), it could account for the observation that not every available nerve terminal sprouts following partial denervation or poisoning with synaptic blocking agents. Formamide exerts a direct blocking effect on the muscle contractile mechanism that is in some way related to the regulation of internal calcium stores (Escalona de Motta, et al., 1982). This form of contractile inactivity may therefore inhibit some calcium-dependent process that in normally active muscle might prevent the appearance of a sprout-promoting factor.

If muscle fiber contractile inactivity alone is the source of a sprout-promoting factor, then a uniform contraction block should have produced extensive terminal sprouting. However, the sprouting response was relatively small and varied, with no apparent pattern of sprouting nerve terminals even after 6 weeks of maintained inactivity. This suggests that even under these well-controlled conditions, many nerve terminals still did not sprout even though they were on or immediately adjacent to chronically inactive muscle fibers and presumably had been exposed to the putative sprout-promoting factor. In this context, the efficacy of any sprout-promoting factor, while potent in poisoned muscles, may be reduced or even inhibited by the presence of functional synaptic transmission and/or muscle action potentials in formamide-blocked muscles. This hypothesis is further supported by our most recent preliminary observations, which show that chronic block of synaptic transmission in $\mathrm{CP}$ muscles by $\alpha$-bungarotoxin produces significantly higher frequencies of terminal sprouting ( $52 \%$ at 6 weeks inactivity) than that promoted by formamide exposure ( $21 \%$ at 6 weeks inactivity) after comparable periods of time (Wines et al., 1987).

We performed several control experiments to assess whether other variables were also affecting the nerve terminal's sprouting response to inactivity. One possibility tested was that formamide itself could have a direct inhibitory effect by reducing or eliminating the production of a sprout-promoting factor or by negating the nerve terminal's ability to respond to this factor. A strong inhibitory effect of formamide probably can be ruled out since prolific sprouting still occurred in partially denervated $\mathrm{CP}$ muscles even when chronically treated with formamide; however, one must consider that these results may be somewhat misleading as this sprouting may have been due to a cue associated with partial denervation (Brown et al., 1981; Brown, 1984) that overrides any inhibitory effect of formamide. Conversely, formamide treatment itself may directly stimulate nerve terminals to sprout. This was particularly difficult to control for since it was not possible to use our standard formamide paradigm without blocking muscle contractions. However, when we altered this regimen to allow for prolonged cxposurc to formamide in a manner that did not block contractions, only very minimal sprouting resulted. This suggests that repeated treatments with formamide were not, in and of themselves, a potent stimulus to terminal sprouting.

The further possibility exists that chronic formamide treatment produced terminal sprouting by an indirect means. For example, these treatments might have caused irritation and inflammatory changes in the membranes of treated muscle fibers which eventually caused sprouting. Analogous changes have been noted in inactive muscle (Lomo and Westgaard, 1976) or even in normal muscles following the application of nerve fragments or silk thread (Jones and Vrbova, 1974). This suggests that inflammation or local irritation might induce terminal sprouting. Although our short-term physiological data and morphological observations suggest that formamide exposure is not deleterious to either neurons or muscle fibers, the possibility still exists that prolonged exposure to formamide may induce cellular changes that eventually manifest as sprouting. This possibility can only be ruled out by future experiments in which the presence or absence of nerve terminal sprouting are correlated with the physiological characteristics of identified nerve terminals.

In summary, 2 major points arise from these studies. We have demonstrated, for the first time, that it is possible to produce chronic muscle fiber contractile inactivity in vivo by selectively inhibiting only the mechanical component of the twitch without significantly altering the electrophysiological properties of the motor axon, neuromuscular junction, or the associated muscle fiber. Second, and perhaps more importantly, these studies have ascertained that muscle fiber contractile inactivity by itself does have some influence over the induction of sprouting from motor nerve terminals; however, the efficacy of this cue appears to be moderate. This may further indicate that the integrity of other physiological parameters, such as synaptic transmission or muscle fiber electrical activity, are also important in the regulation of motor neuron sprouting.

\section{References}

Barker, D., and M. C. Ip (1966) Sprouting and degeneration of mammalian motor axons in normal and de-afferentated skeletal muscle. Proc. R. Soc. London [Biol.] 163: 538-553.

Betz, W. J., J. H. Caldwell, and R. R. Ribchester (1980) Sprouting of active nerve terminals in partially inactive muscles of the rat. J. Physiol. (Lond.) 303: 281-297.

Brown, M. C. (1984) Sprouting of motor nerves in adult muscles: A recapitulation of ontogeny. Trends Neurosci. 7: 10-14.

Brown, M. C., and R. L. Holland (1979) A central role for denervated tissues in causing nerve sprouting. Nature 282: 724-726.

Brown, M. C., and R. Ironton (1977) Motor neuron sprouting induced by prolonged tetrodotoxin block of nerve action potentials. Nature 265: 459-461.

Brown, M. C., G. M. Goodwin, and R. Ironton (1977) Prevention of motor nerve sprouting in botulinum toxin poisoned mouse muscles by direct stimulation of the muscle. Proc. Physiol. Soc. 267: 42$43 \mathrm{P}$.

Brown, M. C., R. L. Holland, W. G. Hopkins, and R. J. Keynes (1980a) An assessment of the spread of the signal for terminal sprouting within and between muscles. Brain Res. 210: 145-151.

Brown, M. C., R. L. Holland, and R. Ironton (1980b) Nodal and terminal sprouting from motor nerves in fast and slow muscles of the mouse. J. Physiol. (Lond.) 306: 493-510.

Brown, M. C., R. L. Holland, and W. G. Hopkins (1981) Motor nerve sprouting. Annu. Rev. Neurosci. 4: 17-42.

Coers, C., N. Telerman-Toppet, and J.-M. Gerard (1973) Terminal innervation ratio in neuromuscular disease. II. Disorders of lower motor neuron, peripheral nerve, and muscle. Arch. Neurol. 29:215222.

Duchen, L. W. (1970) Hereditary motor end-plate disease in the mouse: Light and electron microscopic studies. J. Neurol. Neurosurg. Psychiatry $33: 238-250$.

Duchen, L. W., and S. J. Strich (1968) The effects of botulinum toxin on the pattern of innervation of skeletal muscle in mouse. Q. J. Exp. Physiol. 53: 84-89.

Duchen, L. W., and D. A. Tonge (1973) The effects of tetanus toxin on ncuromuscular transmission and on the morphology of motor endplates in slow and fast skeletal muscle of the mouse. J. Physiol. (Lond.) 228: 157-172.

Edds, M. V. (1950) Collateral regeneration of residual motor axons in partially denervated muscles. J. Exp. Zool. 113: 517-537.

Edds, M. V. (1953) Collateral nerve regeneration. Q. Rev. Biol. 28: 260-276.

Eldridge, L., M. Liebhold, and J. H. Steinbach (1981) Alterations in cat skeletal neuromuscular junctions following prolonged inactivity. J. Physiol. (Lond.) 313: 529-545.

Escalona de Motta, G., F. Cordoba, M. de Leon, and J. del Castillo 
(1982) Inhibitory action of high formamide concentrations on excitation-contraction coupling in skeletal muscle. J. Neurosci. Res. 7: 163-178.

Grinnell, A. D., and A. A. Herrera (1981) Specificity and plasticity of neuromuscular connections: Long-term regulation of motoneuron function. Prog. Neurobiol. 17: 203-282.

Gurney, M. E. (1984) Suppression of sprouting at the neuromuscular junction by immune sera. Nature $307: 546-548$.

Gurney, M. E., B. R. Apatoff, and S. P. Heinrich (1986) Suppression of terminal axonal sprouting at the neuromuscular junction by monoclonal antibodies against a muscle-derived antigen of 56,000 daltons. J. Cell Biol. 102: 2264-2272.

Harris, J. B., and R. R. Ribchester (1979) The relationship between end-plate size and transmitter release in normal and dystrophic muscles of the mouse. J. Physiol. (Lond.) 296: 245-265.

Herrera, A. A. (1984) Polyneuronal innervation and quantal transmitter release in formamide-treated frog sartorius muscles. J. Physiol. (Lond.) 355: 267-280.

Hoffman, H. (1950) Local re-innervation in partially denervated muscle: A histo-physiological study. Aust. J. Exp. Biol. Med. Sci. 28: $383-$ 397.

Holland, R. L., and M. C. Brown (1980) Postsynaptic transmission block can cause terminal sprouting of a motor nerve. Science 207 . 649-651.

Holland, R. L., and M. C. Brown (1981) Nerve growth in botulinum toxin poisoned muscles. Neuroscience 6: 1167-1179.

Jones, R., and G. Vrbova (1974) Two factors responsible for the development of denervation hypersensitivity. J. Physiol. (Lond.) 236: 517-538.

Karnovsky, M. J. (1964) The localization of cholinesterase activity in the rat cardiac muscle by electron microscopy. J. Cell Biol. 23: 217232.

Letinsky, M. S. (1983) Staining normal and experimental motor nerve terminals with tetrazolium salts. Stain Tech. 58: 21-27.

Letinsky, M. S., and P. A. DeCino (1980) Histological staining of preand postsynaptic components of amphibian neuromuscular junctions. J. Neurocytol. 9: 305-320.

Letinsky, M. S., and K. Morrison-Graham (1980) Structure of developing frog neuromuscular junctions. J. Neurocytol. 9: 321-342.

Letinsky, M. S., K. H. Fischbeck, and U. J. McMahan (1976) Precision of reinnervation of original postsynaptic sites in frog muscle after a nerve crush. J. Neurocytol. 5: 691-718.

Linden, D. C., and M. S. Letinsky (1983) Correlated nerve and muscle differentiation in the bull frog cutaneous pectoris. In The Physiology of Excitable Cells, pp. 423-433, Liss, New York.
Linden, D. C., and M. S. Letinsky (1988) Correlated muscle and nerve development in the bullfrog cutaneous pectoris. Exp. Neurol. 99: 709734.

Lomo, T., and R. H. Westgaard (1976) Control of ACh sensitivity by muscle activity in the rat. Cold Spring Harbor Symp. Quant. Biol. 40: 263-274.

McMahan, U. J., D. R. Edgington, and D. P. Kuffler (1980) Factors that influence regeneration of the neuromuscular junction. J. Exp. Biol. 89: 31-42.

Morrison-Graham, K. (1983) An anatomical and electrophysiological study of synapse elimination at the developing frog neuromuscular junction. Dev. Biol. 99: 298-311.

O'Brien, R. A. D., A. J. S. Ostberg, and G. Vrbova (1978) Observations on the elimination of polyneuronal innervation in developing mammalian skeletal muscle. J. Physiol. (Lond.) 282: 571-582.

Pestronk, A., and D. Drachman (1978) Motor nerve sprouting and acetylcholine receptors. Science 199: 1223-1225.

Rowe, K. W. D., and G. Goldspink (1969) Muscle fiber growth in five different muscles in both sexes of mice. II. Dystrophic mice. J. Anat. 104: 531 .

Slack, J. R., and S. Pockett (1981) Terminal sprouting of motoneurons is a local response to a local stimulus. Brain Res. 217: 368-374.

Slack, J. R., and S. Pockett (1982) Motor neurotrophic factor in denervated adult skeletal muscle. Brain Res. 247: 138-140.

Slack, J. R., W. G. Hopkins, and S. Pockett (1983) Evidence for a motor nerve growth factor. Muscle Nerve 6: 243-252.

Tal, M., and S. Rotshenker (1984) Sprouting and synapse formation produced by carbocaine. J. Neurosci. 4: 458-463.

Tuffery, A. R. (1971) Growth and degeneration of motor endplates in normal cat hind limb muscles. J. Anat. 110: 221-247.

Wernig, A., and A. A. Herrera (1986) Sprouting and remodelling at the nerve-muscle junction. Prog. Neurobiol. 27: 251-291.

Wernig, A., M. Pecot-Dechavassine, and H. Stover (1980) Sprouting and regression of the nerve at the frog neuromuscular junction in normal conditions and after prolonged paralysis with curare. J. Neurocytol. 9: 277-303.

Wines, M. M., S. A. Spector, S. P. Carter, M. L. TeVrucht, and M. S. Letinsky (1985) The role of muscle fiber inactivity on motoneuron sprouting. Soc. Neurosci. Abstr. 11: 916.

Wines, M. M., D. G. Garrett, and M. S. Letinsky (1987) The role of contractile activity and synaptic function in motor neuron sprouting. Soc. Neurosci. Abstr. 13: 1392.

Yao, Y. M., and U. J. McMahan (1985) Factors that influence the maintenance of regenerating axon terminals at neuromuscular junctions. Soc. Neurosci. Abstr. 11: 947. 